\title{
Inhibition of return revisited: Localized inhibition on top of a pervasive bias
}

\author{
Benchi Wang ${ }^{1,2} \cdot$ Chuyao Yan ${ }^{1} \cdot$ Raymond M. Klein ${ }^{3} \cdot$ Zhiguo Wang $^{1}$
}

Published online: 15 December 2017

(C) Psychonomic Society, Inc. 2017

\begin{abstract}
An inhibitory after-effect of attention, frequently referred to as inhibition of return (IOR), operates at a previously attended location to discourage perseverative orienting. Using the classic cueing task, previous work has shown that IOR is not restricted to a previously attended location, but rather spreads to adjacent visual space in a graded manner. The present study expands on this earlier work by exploring a wider visual region and a broader range of cue-target onset asynchronies (CTOAs) to characterize the temporal dynamics of the IOR gradient. The results reveal that the magnitude of IOR generated by cueing decreases exponentially as the CTOA increases. The width of the IOR gradient first increases and then decreases, with a temporal profile that is well captured by an alpha function. Importantly, the present study reveals that in addition to its rapidly decaying local properties, cue-induced IOR can include a pervasive inhibitory bias, which remains relatively stable across IOR's lifetime.
\end{abstract}

Keywords Inhibition of return $\cdot$ Spatial attention $\cdot$ Orienting $\cdot$ Cueing task

\section{Introduction}

After attention has been exogenously oriented to a location, processing of stimuli at this location is first facilitated and then inhibited. The latter inhibitory aftereffect of attention, frequently referred to as inhibition of return (IOR), was first thoroughly explored by Posner and colleagues with a spatial cueing task (Cohen, 1981; Posner \& Cohen, 1984; Posner, Cohen, \& Rafal, 1982; Posner, Rafal, Choate, \& Vaughan, 1985). In the prototypical cuing experiment, IOR is operationalized as the difference in response time to targets presented at the cued location relative to targets at the same eccentricity in the opposite visual field. Follow-up studies have explored a multitude of properties of IOR (spatiotopic and object-based

Electronic supplementary material The online version of this article (https://doi.org/10.3758/s13423-017-1410-9) contains supplementary material, which is available to authorized users.

Zhiguo Wang

z.wang@hznu.edu.cn

1 Center for Cognition and Brain Disorders, Hangzhou Normal University, 2138 Yuhangtang Rd, Hangzhou 311121, China

2 Department of Experimental and Applied Psychology, Vrije Universiteit Amsterdam, Amsterdam, The Netherlands

3 Department of Psychology and Neuroscience, Dalhousie University, Halifax, Canada coding, relation to the oculomotor system, and time-course, etc.; for a review, see Klein, 2000), including robust empirical evidence showing that IOR can last for up to 3 seconds (e.g., Samuel \& Kat, 2003). It is now widely recognized that IOR maximizes the sampling of visual information (Posner \& Cohen, 1984) and facilitates visual foraging (e.g., Klein, 1988; Klein \& Macinnes, 1999; for a review, see Wang \& Klein, 2010).

Ever since its discovery, IOR has been an indispensable component of salience-based models of attention (e.g., Itti \& Koch, 2001; Koch \& Ullman, 1985). However, computational theories frequently overlook the fact that IOR is not restricted to a previously attended location, but rather spreads to adjacent space (e.g., Bennett \& Pratt, 2001; Berlucchi, Tassinari, Marzi, \& Di Stefano, 1989; Birmingham, Visser, Snyder, \& Kingstone, 2007; Christie, Hilchey, \& Klein, 2013; Dorris, Taylor, Klein, \& Munoz, 1999; Langley, Gayzur, Saville, Morlock, \& Bagne, 2011; Maylor \& Hockey, 1985; Taylor, Chan, Bennett, \& Pratt, 2015). For instance, Bennett and Pratt (2001) asked their participants to make a button response to targets preceded by an abrupt onset cue. The target appeared at one of 441 locations that covered a $21^{\circ} \times 21^{\circ}$ region, whereas the cue was always centered at one of the four quadrants of this region. IOR manifested in prolonged response times (RTs) to targets at the cued location and, importantly, RTs decreased as the cue-target distance increased, showing a clear spatial gradient for IOR. Other work suggests that this IOR gradient 
is unaffected by response modality (manual or saccadic; Christie et al., 2013; Wang et al., 2014) and can also be observed at previously foveated locations (Vaughan, 1984; Jayaraman, Klein, Hilchey, Patil, \& Mishra, 2015). ${ }^{1}$

In cueing tasks, the magnitude of IOR reaches its maximum about 300-500 ms following cue onset, and then steadily decreases (for reviews, see Klein, 2000; Samuel \& Kat, 2003). However, we have limited knowledge of how the spatial gradient of IOR evolves over time because the time course of IOR is typically examined only at the cued location (for exceptions to this generalization, see Samuel \& Kat, 2003; Taylor et al., 2015; Vaughan, 1984). Here we highlight studies that have examined this issue using manual responses. A coarse characterization of the IOR gradient was performed in Samuel and Kat (2003) by presenting the target $0^{\circ}, 90^{\circ}$, or $180^{\circ}$ (polar angle) away from the cue. This study examined cue-target onset asynchronies (CTOAs) ranging between 0 and 3,200 $\mathrm{ms}$, and clear IOR gradients were observed only for CTOAs below $1 \mathrm{~s}$. A recent study by Taylor et al. (2015) examined the gradient of IOR on a finer scale, but with a narrower range of CTOAs (100-800 ms). The IOR gradient was found to reach a plateau around $400 \mathrm{~ms}$ after cue onset. How the gradient evolves later on remains unclear primarily because of the spatial or temporal limits highlighted in Table 1.

The studies discussed above (Bennett \& Pratt, 2001; Samuel \& Kat, 2003; Taylor et al., 2015) give us a glimpse into the IOR gradient and how it evolves over time. However, considering the parametric (see Table 1) and methodological limitations of these studies - which are discussed next - caution must be taken when interpreting these findings. Firstly, Bennett and Pratt (2001) used a constant CTOA (800 ms) so no information about time-course was provided. Secondly, although the task used by Taylor et al. (2015) allowed for an examination of the IOR gradient over time, the longest CTOA tested was only $800 \mathrm{~ms}$. Because IOR can last for at least $3 \mathrm{~s}$ (e.g., Samuel \& Kat, 2003), CTOAs above $800 \mathrm{~ms}$ are critical in characterizing the time-course of the IOR gradient. Finally, the study by Samuel and Kat (2003) examined a good range of CTOAs (0-3,000 ms), however, only one eccentricity was examined and the cue-target (angular) separations $\left(0^{\circ}, 90^{\circ}\right.$, and $180^{\circ}$ ) were too coarse to accurately reveal the IOR gradient.

The present study was designed to reveal how the IOR gradient evolves over time, with reasonably fine spatial resolution and broad temporal coverage. We highlight three methodological departures from previous work. First, the IOR gradient was probed within a large visual region $\left(\right.$ diameter $=35^{\circ}$ ). Second, we used a broad range of CTOAs $(100-3,600 \mathrm{~ms})$ that were randomly intermixed within a block of trials. Third,

\footnotetext{
${ }^{1}$ The present experiment and discussion focus on IOR effects in cueing tasks that specifically require the participants to avoid gaze shifts.
}

IOR effects were not merely inferred from changes in raw RTs (as in Bennett \& Pratt, 2001 and Taylor et al., 2015), but were quantified by subtracting RTs to targets presented at uncued control locations.

\section{Method}

The present research was approved by the Institutional Review Board of Center for Cognition and Brain Disorders at Hangzhou Normal University, and all participants gave written informed consent.

\section{Participants}

Twelve naïve volunteers (five females, mean age: 20.8 years) from the local community took part in this experiment. All of them were right-handed and reported normal or corrected-tonormal visual acuity. They were paid 40 Yuan per hour.

\section{Apparatus and stimuli}

Participants were tested in a dimly lit laboratory. Visual stimuli were presented on three 19-in. CRT monitors (Samsung SyncMater 997MB) at a viewing distance of $63 \mathrm{~cm}$ (maintained by using a chinrest). The experimental script, written in Python, controlled a Windows 7 PC that presented stimuli on the monitors and registered responses on a keyboard. The monitors sat side by side with a $7.5^{\circ}$ gap separating the visible regions of adjacent monitors. The maximum luminance of the monitors was $124 \mathrm{~cd} / \mathrm{m}^{2}$, whereas the minimum luminance was $2.22 \mathrm{~cd} / \mathrm{m}^{2}, 2.26 \mathrm{~cd} / \mathrm{m}^{2}$, and $2.24 \mathrm{~cd} / \mathrm{m}^{2}$, respectively. The central monitor was used to present a fixation stimulus only, and the side monitors were used to present the cues and targets. As illustrated in Fig. 1, the side monitors were tilted so as to maintain roughly the same viewing distance for all stimuli. To ensure the participants maintained fixation, an EyeLink ${ }^{\circledR} 1000$ eye-tracker was used to monitor the participant's gaze direction during testing. The spatial accuracy of this eye tracker was reported to be $0.25^{\circ}$ or better; the sampling rate was set to $500 \mathrm{~Hz}$.

The fixation stimulus was a white cross measuring $1^{\circ} \times 1^{\circ}$; the cue was an empty white square measuring $1^{\circ} \times 1^{\circ}$; and the target was a filled white disk, with a radius of $0.5^{\circ}$. All stimuli were presented against a black background (Weber contrast $=$ 97.5).

The fixation was presented at the center of the central monitor, while the target always appeared on the horizontal meridian, $49.5^{\circ}$ left or right to the fixation (i.e., at the center of the side monitors). The cue was presented at one of 11 possible locations on the diagonals of the side monitors (see Fig. 1a). When the cue and target appeared on the same monitor, the distance between them could be $0-17.5^{\circ}$, in steps of $3.5^{\circ}$. 
Table 1 The methodological properties of previous studies and the present study

\begin{tabular}{lllll}
\hline Study & Samuel \& Kat 2003 & Bennet \& Pratt 2001 & Taylor et al. 2015 & Present study \\
\hline Grain of C-T separations & Low & Very high & Very high & High \\
Cue eccentricities & $6.8^{\circ}$ & $8.4^{\circ}$ & $8.5^{\circ}$ & $32-67^{\circ}$ \\
Target eccentricities & $6.8^{\circ}$ & $1-14.1^{\circ}$ & $1-14.1^{\circ}$ & $49.5^{\circ}$ \\
Range of gradient & $0-180^{\circ}$ (polar) & $21^{\circ}$ & $21^{\circ}$ & $35^{\circ}$ \\
CTOAs (ms) & $0-3,000$ & 800 & $0-800$ & $100-3,600$ \\
\hline
\end{tabular}

\section{Procedure and design}

The participant's gaze direction was constantly monitored during testing. A self-paced drift check was performed at the beginning of each trial. Then, a fixation cross appeared and remained visible throughout the trial. 1,000 ms after fixation onset, the cue appeared at one of the 42 possible locations for $100 \mathrm{~ms}$. The cue was spatially uninformative and participants were explicitly instructed to ignore it and to maintain fixation. The target was presented $100 \mathrm{~ms}, 400 \mathrm{~ms}, 1,200 \mathrm{~ms}, 2,400 \mathrm{~ms}$, or 3,600 ms after cue onset. The target could appear on the same monitor as the cue or on the opposite monitor. The participants had $1,500 \mathrm{~ms}$ to issue a response, by pressing the ' $z$ ' or '/' keys to targets presented on the left or right monitor, respectively. The next trial began after a randomly jittered (1,000-1,500 ms) inter-trial interval (ITI). Each participant completed a total of 2,940 trials in seven sessions. Warning messages were displayed if the participant's gaze deviated more than $2^{\circ}$ from fixation, or if they pressed the wrong key. Trials with such warning messages were later retested in a random order, until all trials were completed successfully. a

Bennett \& Pratt'2001

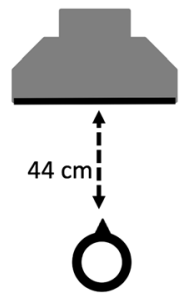

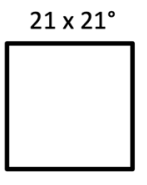
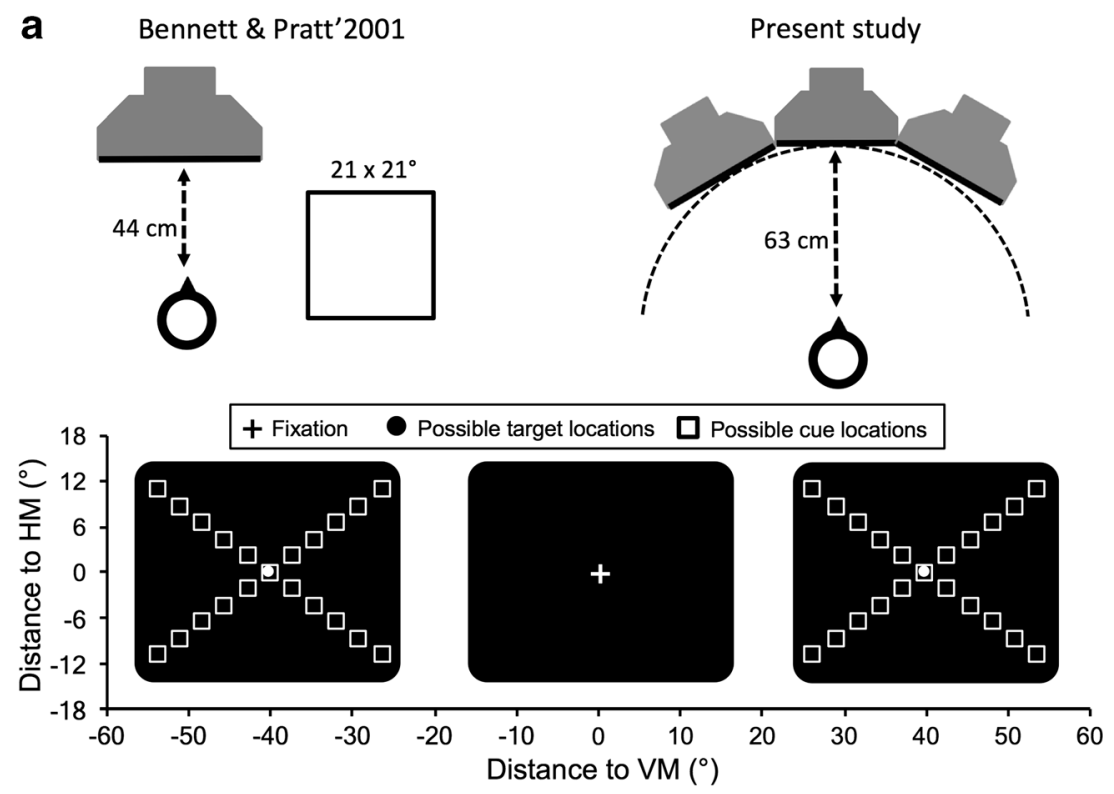

b

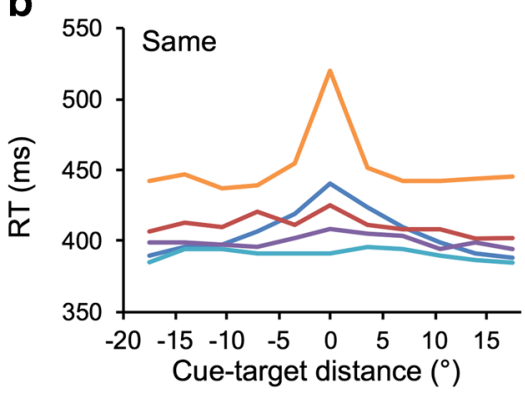

Fig. 1. (A) The three-monitor setup of the present study and possible cue and target locations on the displays. Compared to previous work (e.g., Bennett \& Pratt (2001), the present setup allows probing IOR gradient in a much larger visual region. The present experiment did not use

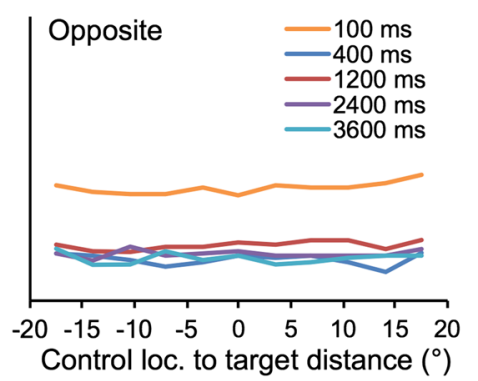

placeholders. HM, horizontal meridian; VM, vertical meridian. (B) Mean RTs to targets in the same and opposite hemifield as the cue. Positive values on the abscissa denote locations more eccentric than the target 
In addition to this experiment, we also report two pilot experiments as Supplementary Material. The display setup of these experiments was largely the same; the CTOAs tested in these two experiments were $100 \mathrm{~ms}, 400 \mathrm{~ms}$, and $1,200 \mathrm{~ms}$, and $800 \mathrm{~ms}, 1,600 \mathrm{~ms}$, and 3,200 ms, respectively. The overall pattern of results obtained from the pilot experiments was consistent with that reported here, providing converging evidence for the conclusions drawn in the present paper.

\section{Results}

Erroneous responses to the target accounted for only $0.68 \%$ of the trials on which fixation was properly maintained. Error rates were slightly higher for targets in the same hemifield $(0.76 \%)$ compared to those in the opposite hemifield $(0.61$ $\%)$ as the cue. This difference, however, was not significant, $\mathrm{t}(11)=1.13, \mathrm{p}=.217, \mathrm{~d}_{\mathrm{z}}=.378$.

To increase statistical power and to minimize the effect of visual fields (upper vs. lower) on RTs, same-monitor cues of the same horizontal position were aggregated. Possible physical differences between the side monitors were controlled for by sorting trials based on whether the target appeared in the same or opposite hemifield as the cue, as in classic cueing tasks. The RTs were then trimmed on a per-experimental condition, per-participant basis. Based on the criteria given in Van Selst and Jolicoeur (1994, Table 4), RTs that were 2.42 SDs above the mean of an experimental condition were excluded from further analysis. This procedure removed only a small portion of the successfully completed trials (3.02\%).

The mean RTs are presented in Fig. 1b, and in Table S1 in the Supplementary Material. A repeated measures ANOVA was performed on the RTs, with variables cueing (same vs. opposite), cue-target distance $\left(-17.5^{\circ}\right.$ to $17.5^{\circ}$, in steps of $3.5^{\circ}$; negative and positive values denote cues less and more eccentric than the target, respectively), and CTOA (100 ms, $400 \mathrm{~ms}$, $1,200 \mathrm{~ms}, 2,400 \mathrm{~ms}$, and 3,600 ms). The results revealed significant main effects for cueing, $F(1,11)=31.61$, $p<.001$, partial $\eta^{2}=.75$, cue-target distance, $\mathrm{F}(10,110)=9.77, \mathrm{p}<$ .001 , partial $\eta^{2}=.47$, and CTOA, $\mathrm{F}(4,44)=45.48, \mathrm{p}<.001$, partial $\eta^{2}=.81$. As clearly shown in Fig. $1 \mathrm{~b}$, RTs were longer for targets in the cued hemifield relative to those in the opposite hemifield, suggesting an overall IOR effect. The RTs were also slower for small cue-target distances, suggesting a clear spatial gradient. RTs were faster for longer CTOAs, indicating that the temporal expectation of the target intensified with time after cue onset (i.e., a foreperiod effect; Niemi \& Naatanen, 1981). Three significant two-way interactions occurred: between cueing and cue-target distance, $F(10,110)=13.49, p$ $<.001$, partial $\eta^{2}=.55$, because the RT gradient was only prominent when targets appeared in the cued hemifield; between cue-target distance and CTOA, F(40, 440) $=2.86, \mathrm{p}<$ .001 , partial $\eta^{2}=.21$, because the RT gradient became less prominent as CTOA increased; and between cueing and CTOA, $\mathrm{F}(4,44)=5.64, \mathrm{p}<.001$, partial $\eta^{2}=.34$, because the overall IOR effect - the RT difference between targets in the cued and opposite hemifield - generally decreased as the CTOA increased. The three-way interaction also reached significance, $\mathrm{F}(40,440)=4.14, \mathrm{p}<.001$, partial $\eta^{2}=.27$.

IOR effects were computed as the RT difference between same-eccentricity targets presented in the cued ("same") and "opposite" hemifield. These IOR scores at different CTOAs are plotted in Fig. 2a-e. The IOR gradients were assumed to be Gaussian shaped and the magnitude of IOR (I) as a function of cue-target distance (d) can be described with the following function:

$I=a^{*} \exp \left(-\pi^{*}\left(0.94^{*}((d-s)) / w\right)^{2}\right)+b$

where $\mathrm{a}$ is the height of the best-fitting Gaussian of the IOR gradient, $\mathrm{w}$ is the diameter at half height (FWHM), s reflects the shift of the peak from the cued location, and $b$ represents a global inhibitory bias.

Equation 1 was fit to the data presented in Fig. 2a-e by minimizing the root mean squared error between the observed and predicted IOR effects. The best-fitting parameters are presented in Table 2. As clearly shown in Fig. 2 f, the IOR effect at the cued location decayed exponentially as the CTOA increased. The spatial extent of the IOR gradient $(w)$, quantified with the FWHM of the best fitting Gaussian function, first increased and then decreased, with a temporal profile that follows the function below (see Fig. 2g):

$w=\max _{-} w^{*}\left(C T O A / t_{-} \text {peak }\right)^{*} \exp \left(1-C T O A / t_{-}\right.$peak $)$

where $t$ peak is the CTOA at which the FWMH reached its maximum (max_w). For the best-fitting curve presented in Fig. 2g, max_w $=19.93^{\circ}$ and $t \_$peak $=1,030 \mathrm{~ms}$. The global inhibitory bias b was relatively stable across the CTOAs tested in the present experiment (see Fig. 2h), with a slope close to zero $(-0.54 \mathrm{~ms} / \mathrm{s})$.

\section{Discussion}

Consistent with previous findings, the present results show that IOR is not restricted to the cued location, but rather has a fairly large spatial gradient (e.g., Bennett \& Pratt, 2001; Wang et al., 2014). Not surprisingly, the magnitude of IOR decreased as the CTOA increased. The width of the IOR gradient, on the other hand, first increased and then decreased, with a temporal profile that followed an alpha function (see Fig. 2g). Perhaps surprisingly, the present results also revealed that IOR begins with a pervasive bias that remains relatively stable across the lifetime of the IOR effect as explored here (see Fig. 2h). These findings suggest that IOR is best 

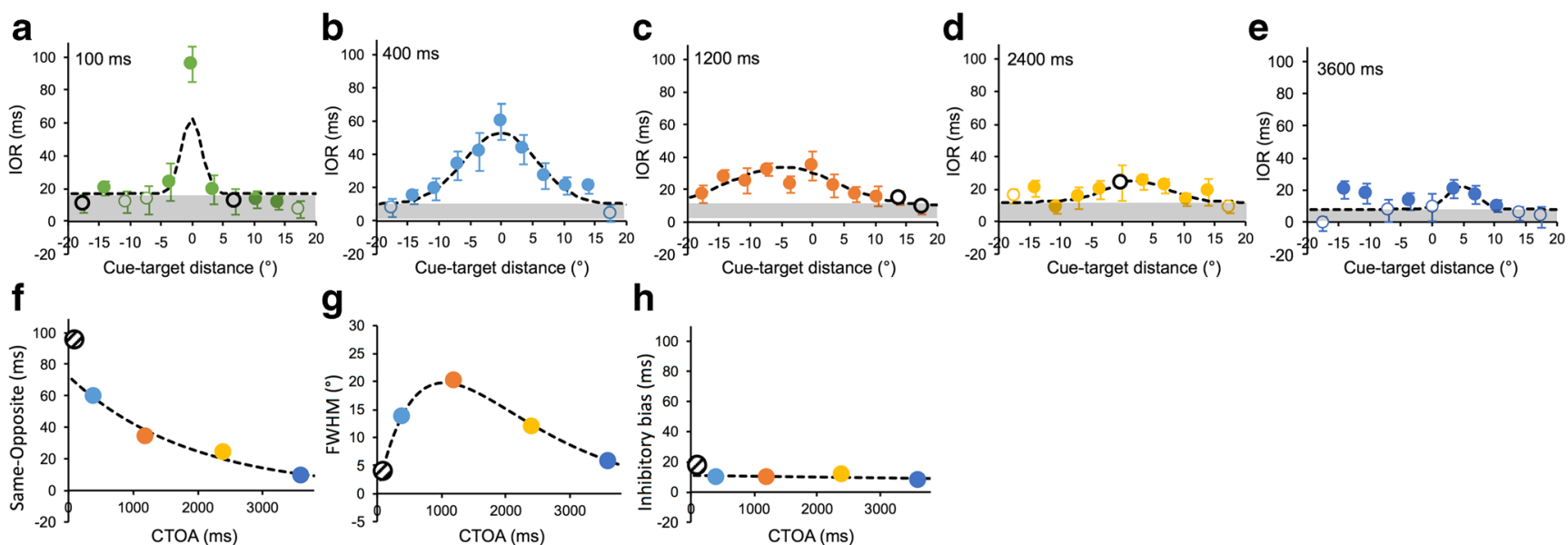

h

Cue-target distance $\left(^{\circ}\right)$

Fig. 2. (A-E) The IOR effects and the best fitting Gaussians (dashed curves) at different CTOAs. Filled circles denote significant IOR effects ( $p<.05,2$-tailed) and empty black circles denote marginally significant IOR effects ( $<<.10,2$-tailed); for illustration purpose only, all $p$-values are uncorrected. The global inhibitory "bias" component from the best fitting function is marked by gray strips. Error bars denote \pm 1 SEM. (F) IOR effects at the cued location decayed exponentially as the CTOA increased. (G) The FWHMs of the best fitting Gaussians to the IOR

described as localized inhibition on top of a pervasive inhibitory bias.

One observation of the present study was that, when the CTOA was only $100 \mathrm{~ms}$, no RT benefit was observed for targets presented at the cued location; instead, a strong RT cost was observed. We note that, in previous works, RT benefits are not reliably observed when the CTOA is short (for a summary of relevant findings, see Collie, Maruff, Yucel, Danckert, \& Currie, 2000). For instance, Collie et al. (2000) showed that peripheral cues only led to RT benefits when they were temporally overlapping with the target. When the target was presented immediately after the cue, no RT benefit was observed. We believe the strong RT cost $(\mathrm{M}=100 \mathrm{~ms})$ at the cued location at the $100 \mathrm{~ms}$ CTOA cannot be attributed primarily to IOR. One possibility is that when the target appeared immediately after the cue, forward masking may have led to a detection cost for the target. Such a low-level masking effect cannot be the whole story, however, because in a physically

Table 2 Best-fitting parameters * for the IOR effects at different CTOAs and corresponding $\mathrm{R}^{2}$

\begin{tabular}{llllll}
\hline CTOA $(\mathrm{ms})$ & $\mathrm{a}$ & $\mathrm{b}$ & $\mathrm{s}$ & $\mathrm{w}$ & $\mathrm{R}^{2}$ \\
\hline 100 & 45.49 & 16.99 & 0.10 & 3.89 & 0.79 \\
400 & 43.04 & 10.16 & -0.14 & 13.83 & 0.92 \\
1,200 & 23.56 & 10.22 & -4.87 & 20.22 & 0.87 \\
2,400 & 13.04 & 12.06 & 2.42 & 11.94 & 0.75 \\
3,600 & 14.37 & 7.77 & 4.79 & 5.75 & 0.40 \\
\hline
\end{tabular}

*a is the height of the best-fitting Gaussian of the IOR gradient, b represents a global inhibitory bias, $\mathrm{s}$ reflects the shift of the peak from the cued location, and $\mathrm{w}$ is the diameter at half height (FWHM) gradients. The temporal profile of the FWHMs was precisely described by an alpha function (see text for details). (H) The pervasive inhibitory bias evoked by IOR (i.e., the gray strips in A-E) remains stable across the CTOAs tested in the present experiment. In (F-H), the $100 \mathrm{~ms}$ CTOA (circle with strips) was excluded from the fittings because the estimated IOR effect at this CTOA was likely conflated by forward masking (see the Discussion section)

identical condition of a pilot experiment with only 3 CTOAs (see Supplementary Material) we found costs that were very much smaller than in the present one. Instead of (or in addition to) the proposed forward masking account, we believe that our participants may have been relying on the detection of two peripheral stimuli (cue and target) as a diagnostic that the target had been presented (see Lupiañez \& Weaver, 1998). Such a diagnostic would work so long at the cue and target were in different locations or there was a temporal gap between them. When such a diagnostic was used, an RT delay, unrelated to IOR, would result when the cue and target were in the same location and the CTOA was $100 \mathrm{~ms}$. We believe that the reduction of costs in this unique condition from $100 \mathrm{~ms}$ in the main experiment to $30 \mathrm{~ms}$ in the pilot experiment might be attributed to the higher frequency of no-gap (100 ms CTOA) trials in the pilot experiment ( $33 \%)$ compared to the main experiment $(20 \%)$.

Previous work has shown that IOR may not spread beyond a placeholder (e.g., Taylor et al., 2015). Because the participants might have been seeing each monitor as an "object", one may suggest that the change in the IOR gradient might be inhibition spreading from the cued location to the cued object (monitor) as the CTOA increased. We note that object-based IOR, first discovered by Tipper et al. (1994), has not always been observed in static displays (for a review, see Reppa, Schmidt, \& Leek, 2012). In a study examining its boundary conditions, List and Robertson (2007) observed object-based IOR only when a back-to-centre cue was present and when the cue- (peripheral or central) to-target interval was approximately between 600 and 1,200 ms. The present study had no backto-center cue and significant IOR effects were observed at much longer CTOAs. Although we could not rule out this 
possibility, it is unlikely that our findings were conflated by object-based IOR.

Logically speaking, RTs to a target are more closely related to target properties than to cue properties. In our study, possible target locations were fixed at the center of the left- and right-side monitors. With this method estimation of the IOR effect would not be conflated by the effects of the position of the target (such as eccentricity). It is worth noting that the target eccentricity effect was evident in Bennett and Pratt (2001), and was not assessed in Taylor et al. (2015). However, without a target that was $90^{\circ}$ (polar angle) away from the cue (as in Samuel \& Kat, 2003), we could not determine if the inhibition observed for far cue-target distances was actually facilitation in the opposite (uncued) hemifield. This issue warrants further investigation.

To function as an effective inhibitory mechanism that discourages perseverative orienting, IOR needs to last for a relatively long period. If IOR had a very brief life span, its usefulness in visual foraging tasks would be fairly limited as these tasks typically require a few seconds to complete. The present finding that the IOR gradient is best depicted as localized inhibition superimposed on a pervasive inhibitory bias prompts reconsideration of the ecological explanation of IOR. While the present findings do not challenge the idea that IOR encourages orienting towards novelty, they do suggest that IOR may operate in two different modes. As clearly shown in Fig. 2, IOR is initially strong and has a sharp tuning; later on, as the magnitude of IOR quickly decreases, although the extent of the IOR gradient also reduces, the pervasive inhibitory bias dominates. Based on these observations, we suggest that IOR operates on previously attended locations during the initial phase, and functions as a pervasive bias later on.

Author notes This project was supported by a National Natural Science Foundation of China (NSFC) grant (31371133) to ZW, a Natural Sciences and Engineering Research Council of Canada (NSERC) Discovery grant (9948-2011) to RMK, and a China Scholarship Council (CSC) scholarship (201508330313) to BW.

\section{References}

Bennett, P., \& Pratt, J. (2001). The spatial distribution of inhibition of return. Psychological Science, 12(1), 76-80. doi:https://doi.org/10. 1111/1467-9280.00313

Berlucchi, G., Tassinari, G., Marzi, C. A., \& Di Stefano, M. (1989). Spatial distribution of the inhibitory effect of peripheral noninformative cues on simple reaction time to non-fixated visual targets. Neuropsychologia, 27(2), 201-221.

Birmingham, E., Visser, T. A., Snyder, J. J., \& Kingstone, A. (2007). Inhibition of return: Unraveling a paradox. Psychonomic Bulletin \& Review, 14(5), 957-963.

Christie, J., Hilchey, M. D., \& Klein, R. M. (2013). Inhibition of return is at the midpoint of simultaneous cues. Attention, Perception, \& Psychophysics, 75(8), 1610-1618.
Cohen, Y. A. (1981). Internal and External Control of Visual Orienting. University of Oregon.

Collie, A., Maruff, P., Yucel, M., Danckert, J., \& Currie, J. (2000). Spatiotemporal distribution of facilitation and inhibition of return arising from the reflexive orienting of covert attention. Journal of Experimental Psychology: Human Perception and Performance, 26(6), 1733-1745. doi:https://doi.org/10.1037/0096-1523.26.6. 1733

Dorris, M. C., Taylor, T. L., Klein, R. M., \& Munoz, D. P. (1999). Influence of previous visual stimulus or saccade on saccadic reaction times in monkey. Journal of Neurophysiology, 81(5), 2429-2436.

Itti, L., \& Koch, C. (2001). Computational modelling of visual attention. Nature Reviews Neuroscience, 2(3), 194-203. doi:https://doi.org/ $10.1038 / 3505850$

Jayaraman, S., Klein, R. M., Hilchey, M. D., Patil, G. S., \& Mishra, R. K. (2015). Spatial gradients of oculomotor inhibition of return in deaf and normal adults. Experimental Brain Research, 1-8. doi:https:// doi.org/10.1007/s00221-015-4439-x

Klein, R. (1988). Inhibitory tagging system facilitates visual search. Nature, 334(6181), 430-431. doi:https://doi.org/10.1038/334430a0

Klein, R. (2000). Inhibition of return. Trends in Cognitive Sciences, 4(4), 138-147. doi:https://doi.org/10.1016/S1364-6613(00)01452-2

Klein, R. M., \& Macinnes, J. W. (1999). Inhibition of return is a foraging facilitator in visual search. Psychological Science, 10(4), 346-352. doi:https://doi.org/10.1111/1467-9280.00166

Koch, C., \& Ullman, S. (1985). Shifts in selective visual attention: Towards the underlying neural circuitry. Human Neurobiology, 4(4), 219-227.

Langley, L. K., Gayzur, N. D., Saville, A. L., Morlock, S. L., \& Bagne, A. G. (2011). Spatial distribution of attentional inhibition is not altered in healthy aging. Attention, Perception, \& Psychophysics, 73(3), 766-783.

List, A., \& Robertson, L. C. (2007). Inhibition of return and object-based attentional selection. Journal of Experimental Psychology: Human Perception and Performance, 33(6), 1322-1334.

Lupiañez, J., \& Weaver, B. (1998). On the time course of exogenous cueing effects: A commentary on Tassinari et al.(1994). Vision Research, 38, 1621-1624.

Maylor, E. A., \& Hockey, R. (1985). Inhibitory component of externally controlled covert orienting in visual space. Journal of Experimental Psychology: Human Perception and Performance, 11(6), 777-787.

Niemi, P., \& Naatanen, R. (1981). Forepriod and simple reaction-time. Psychological Bulletin, 89(1), 133-162. doi:https://doi.org/10. 1037//0033-2909.89.1.133

Posner, M. I., \& Cohen, Y. (1984). Components of visual orienting. In H. Bouma \& D. G. Bouwhuis (Eds.), Attention and Performance X: Control of language processes (Vol. 10, pp. 531-556). Hillsdale, NJ: Erlbaum.

Posner, M. I., Cohen, Y., \& Rafal, R. D. (1982). Neural systems control of spatial orienting. Philosophical Transactions of the Royal Society of London. B, Biological Sciences, 298(1089), 187-198.

Posner, M. I., Rafal, R. D., Choate, L. S., \& Vaughan, J. (1985). Inhibition of return: Neural basis and function. Cognitive Neuropsychology, 2(3), 211-228. doi:https://doi.org/10.1080/02643298508252866

Reppa, I., Schmidt, W. C., \& Leek, E. C. (2012). Successes and failures in producing attentional object-based cueing effects. Attention, Perception \& Psychophysics, 74(1), 43-69. https://doi.org/10. 3758/s13414-011-0211-x

Samuel, A., \& Kat, D. (2003). Inhibition of return: A graphical metaanalysis of its time course and an empirical test of its temporal and spatial properties. Psychonomic Bulletin \& Review, 10(4), 897-906.

Taylor, J. E. T., Chan, D., Bennett, P. J., \& Pratt, J. (2015). Attentional cartography: Mapping the distribution of attention across time and space. Attention, Perception, \& Psychophysics, 77(7), 2240-2246.

Tipper, S. P., Weaver, B., Jerreat, L. M., \& Burak, A. L. (1994). Objectbased and environment-based inhibition of return of visual attention. 
Journal of Experimental Psychology: Human Perception and Performance, 20(3), 478-499.

Van Selst, M., \& Jolicoeur, P. (1994). A solution to the effect of sample size on outlier elimination. The Quarterly Journal of Experimental Psychology Section A: Human Experimental Psychology, 47(3), 631-650. doi:https://doi.org/10.1080/14640749408401131

Vaughan, J. (1984). Saccades directed at previously attended locations in space. Theoretical and Applied Aspects of Eye Movement Research, Selected/Edited Proceedings of the Second European
Conference on Eye Movements (Vol. 22, pp. 143-150). NorthHolland.

Wang, B., Hilchey, M. D., Cao, X., \& Wang, Z. (2014). The spatial distribution of inhibition of return revisited: No difference found between manual and saccadic responses. Neuroscience Letters, 578, 128-132. doi:https://doi.org/10.1016/j.neulet.2014.06.050

Wang, Z., \& Klein, R. M. (2010). Searching for inhibition of return in visual search: A review. Vision Research, 50(2), 220-228. doi: https://doi.org/10.1016/j.visres.2009.11.013 